\title{
Antioxidant capacity of colostrum milk is influenced by maternal diet
}

\author{
A. Navarro-Ruiz ${ }^{1}$, M. T. Hernández-Aguilar ${ }^{2}$, P. Rodríguez-Castellano ${ }^{1}$, A. B. López-Jaén ${ }^{3}$, \\ V. Valls-Bellés ${ }^{3}$ and P. Codoñer-Franch ${ }^{1,3}$ \\ ${ }^{1}$ Department of Pediatrics, Dr. Peset University Hospital, Valencia, Spain, ${ }^{2}$ Arabista Ambrosio Huici Health Centre. \\ Breastfeeding Clinic. Agencia Valenciana de Salud, Departamento 10, Valencia, Spain and ${ }^{3}$ Department of Pediatrics, \\ Obstetrics and Gynecology, University of Valencia, Valencia, Spain
}

Human milk is recognized as the ideal food for the human infant. It contains numerous components that protect the infant from infection and, presumably, diseases in adulthood. Some of the benefits of breastfeeding, such as specific defense mechanisms needed by the infant, have been recently attributed to this antioxidant content. Colostrum offers the most critical protection because the newborn presents an immaturity of the defense systems.

The aim of the present study was to test whether diet of the mothers influence the antioxidant properties of their breast milk. To obtain general information regarding oxidative stress, we measured the antioxidant capacity in breast milk. Total antioxidant capacity is a measure of all of the antioxidants present in the biological fluid considered (in this case, breast milk), and it includes vitamins, enzymatic radical scavenger systems and yet unknown antioxidants.

Fifty four postpartum mothers were included in the study. The inclusion criteria for participants were: mothers without any acute or chronic illness, who were not receiving medications or vitamin supplements, who were non-smokers and did not abuse substances. The women were asked to complete a 3-day dietary record of all the food and beverages consumed (including one Sunday) and a 24-hour recall with composition and portion sizes. Energy and macro and micronutrient intake, were calculated using the validated software Food and Health 0698.046 (BiatASDE General Médica Farmacéutica, Valencia, Spain). Total antioxidant capacity of the mothers' milk was measured using the 2,2'-azinobis (3-ethylbenzothiazoline-6-sulfonic acid) (ABTS) assay (Miller y Rice Evans,1997), modified by Pellegrini ${ }^{(1)}$. Statistical analysis was done by Pearson's correlation coefficient between antioxidant capacity of colostrum and macro and micronutrient intake. We have found a positive correlation between the colostrum antioxidant capacity and the intake of micronutrients: vitamin A $(r=0.348, P=0.032)$, Folic acid $(r=0.381, P=0.001)$ and copper $(r=0.493, P=0.001)$. No correlation was found between colostrum antioxidant capacity and macronutrient intake or energy intake.

Maternal diet, mainly in its micronutrient content, is a factor fundamental to assure antioxidant capacity of breast milk that can be protective to the infant.

1. Pellegrini N, Del Rio D, Colombi B et al. (2003) J Agric Food Chem 51, 260-264. 